\title{
腐殖酸催化合成 1,4-二氢吡啶类化合物
}

\author{
魏振中李江飞王泽云李品华王永秋* \\ (淮北师范大学化学与材料科学学院 淮北 235000)
}

摘要 芳醛、乙酰乙酸乙酯(甲酯)和乙酸铵为原料, 用腐殖酸催化一锅法合成 $1,4-$ 二氢吡啶类化合物. 该法产率较高, 操作简单. 催化剂易于回收再利用，环境友好.

关键词 腐殖酸, 催化, 1,4-二氢吡啶

\section{Synthesis of 1,4-Dihydropyridine Compounds Catalyzed by Humic Acid}

\author{
Wei, Zhenzhong Li, Jiangfei $\quad$ Wang, Zeyun Li, Pinhua $\quad$ Wang, Yongqiu* \\ (Department of Chemistry and Materials Science, Huaibei Normal University, Huaibei 235000)
}

\begin{abstract}
A novel, efficient and straightforward method to synthesize 1,4-dihydropyridine compounds with excellent yields is reported through Hantzsch reaction of aldehydes, ammonium acetate and ethyl acetoacetate or methyl acetoacetate using humic acid as the catalyst. This method has high yield and less pollution. The catalyst is easy to recycle and environment friendly.

Keywords humic acid; catalysis; 1,4-dihydropyridine
\end{abstract}

1,4-二氢吡啶类化合物是一种钻离子通道阻滞剂类 药物, 在 $\mathrm{K}^{+}$和 $\mathrm{Na}^{+}$通道调制中也发挥重要的作用 ${ }^{[1]}$. 如 正在被广泛使用的治疗高血压和心脑血管疾病的尼卡 地平、尼莫地平、非洛地平等药物 ${ }^{[2]}$, 该类化合物还可 以用来治疗阿尔茨海默氏病 ${ }^{[3]}$. 另外作为药物或药物中 间体还具有抗炎 ${ }^{[4]}$ 、抗病毒 ${ }^{[4]}$ 、抗细胞毒性 ${ }^{[5]}$ 、抗惊厥 ${ }^{[6]}$ 、 抗结核病 ${ }^{[7]}$ 、抗血栓形成 ${ }^{[8]}$ 等作用. 因此, 研究该类化合 物的合成方法, 成为广大化学工作人员关注的热点.

最早合成 1,4-二氢吡啶类化合物的方法是 Hantzsch 法，该方法反应时间长，产率低，且氨气容易对环境造 成污染, 不利于工业化生产. 近年来有很多文献从催化 剂、合成手段、合成方法等方面研究报道 1,4-二氢吡啶 类化合物的合成. 催化剂方面有磁性 $\mathrm{Fe}_{3} \mathrm{O}_{4}$ 纳米粒子 ${ }^{[9]}$ 、 纳米 $\mathrm{WO}_{3}$ 负载磺酸 ${ }^{[10]}$ 、脲酶 ${ }^{[11]}$ 、脯氨酸 ${ }^{[12]}$ 、氨基磺 酸 $^{[13]}$ 、碘 ${ }^{[14]} 、 \mathrm{Ce}\left(\mathrm{SO}_{4}\right)_{2} \bullet \mathrm{SiO}_{2}{ }^{[15]} 、 \mathrm{MCM}-41-\mathrm{Ni}$ 复合物 ${ }^{[16]}$ 、 $\mathrm{TBAB}^{[17]} 、 \mathrm{CuI}^{[18]}$ 、离子液体 ${ }^{[19]}$; 合成方法上有 $3+3$ 环 合 ${ }^{[20]} 、 \beta$-环化反应 ${ }^{[21]}$ 等; 合成手段上有超声及超声介

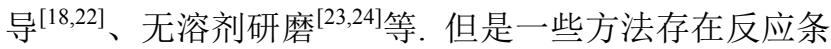
件苛刻、合成催化剂复杂、催化剂污染大、产物后处理 麻烦等缺点, 因此发展一种高效、简单、催化剂可回收 利用的适合于工业化生产 1,4-二氢吡啶类化合物的方法 至关重要.

腐殖酸是自然界中广泛存在的大分子有机物质，广 泛应用于农、林、牧、石油、化工、建材、医药卫生、 环保等各个领域; 作为催化剂或催化剂载体具有廉价易 得、污染小、催化活性较高, 且易于回收利用等优 点 ${ }^{[25,26]}$. 本文以腐殖酸催化 Hantzsch 反应, 以芳醛、乙 酰乙酸乙酯(甲酯)和乙酸铵为原料，一锅法合成了 1,4二氢吡啶类化合物. 方法以 $95 \%$ 乙醇为溶剂, 反应条件 温和且溶剂容易回收; 催化剂不仅无毒、无残留、廉价、 易得，且能很好的回收并连续使用，适合放大生产; 产 品后处理简便只需过滤回收催化剂、尤其是放大生产时 仅需重结晶即可以 $95 \%$ 左右的收率得到产物，整个反应 过程环境十分友好. 合成路线如 Eq. 1 所示.

\footnotetext{
* Corresponding author. E-mail: wyqmsy@163.com

Received December 22, 2016; revised March 13, 2017; published online April 10, 2017.

Project supported by the Open Fund of Anhui Collaborative Innovation Center for Advanced Function Composites (No. 20160804).

安徽省先进功能复合材料协同创新中心开放基金(No. 20160804)资助项目.
} 
$\stackrel{\mathrm{O}}{\stackrel{\mathrm{O}}{\mathrm{N}} \mathrm{R}^{1}}+\mathrm{R}^{2}-\mathrm{CHO}+\mathrm{CH}_{3} \mathrm{COONH}_{4} \underset{\mathrm{CH}_{3} \mathrm{CH}_{2} \mathrm{OH}}{\stackrel{\text { humic acid }}{\longrightarrow}}$<smiles>[R]C(=O)C1=C(C)NC(C)=C(C([R])=O)C1[R]</smiles>

\section{1 结果与讨论}

以 4-甲氧基苯甲醛、乙酰乙酸乙酯和醋酸铵为例, 探究了催化剂用量和溶剂对反应的影响. 结果如表 1 和 表 2 .

表 1 催化剂用量对产率的影响 ${ }^{a}$

Table 1 Effect of catalyst dosage on yield

\begin{tabular}{ccc}
\hline 序号 & 用量 $/ \mathrm{g}$ & 产率/\% \\
\hline 1 & 0.02 & 85 \\
2 & 0.06 & 90 \\
3 & 0.08 & 93 \\
4 & 0.10 & 95 \\
5 & 0.12 & 94.5 \\
6 & 0.15 & 95 \\
\hline
\end{tabular}

${ }^{a} 1.0 \mathrm{mmol} 4$-甲氧基苯甲醛, $2.1 \mathrm{mmol}$ 乙酰乙酸乙酯, $2.0 \mathrm{mmol}$ 醋酸铵, 10 $\mathrm{mL} 95 \%$ 乙醇, 回流 $4.0 \mathrm{~h}$.

表 2 溶剂对反应的影响 ${ }^{a}$

Table 2 Effect of solvent on reaction

\begin{tabular}{llcc}
\hline 序号 & \multicolumn{1}{c}{ 溶剂 } & Time $/ \mathrm{h}$ & 产率/\% \\
\hline 1 & $\mathrm{CH}_{3} \mathrm{CN}$ & 4.0 & 88 \\
2 & $\mathrm{CH}_{2} \mathrm{Cl}_{2}$ & 8.0 & 20 \\
3 & $\mathrm{CHCl}_{3}$ & 8.0 & 35 \\
4 & $\mathrm{THF}$ & 4.0 & 93 \\
5 & $\mathrm{CH}_{3} \mathrm{OH}$ & 5.0 & 84 \\
6 & $\mathrm{C}_{2} \mathrm{H}_{5} \mathrm{OH}$ & 4.0 & 90 \\
7 & $95 \mathrm{C}_{2} \mathrm{H}_{5} \mathrm{OH}$ & 4.0 & 95 \\
8 & $V\left(\mathrm{C}_{2} \mathrm{H}_{5} \mathrm{OH}\right): V\left(\mathrm{H}_{2} \mathrm{O}\right)=1: 1$ & 4.5 & 93 \\
9 & $V\left(\mathrm{C}_{2} \mathrm{H}_{5} \mathrm{OH}\right): V\left(\mathrm{H}_{2} \mathrm{O}\right)=4: 1$ & 4.0 & 95 \\
10 & $\mathrm{H}_{2} \mathrm{O}$ & 8.0 & 40 \\
\hline
\end{tabular}

${ }^{a} 1.0 \mathrm{mmol}$ 4-甲氧基苯甲醛, $2.1 \mathrm{mmol}$ 乙酰乙酸乙酯, $2.0 \mathrm{mmol}$ 醋酸铵, 0.1 $\mathrm{g}$ 腐殖酸, 回流.

由表 1 可以看出, 催化剂的量增加到 $0.10 \mathrm{~g}$ 以上后 对产率影响不大, 因此选用催化剂的用量为 $0.10 \mathrm{~g}$. 由 表 2 可以看出, 在醇、醇水、四氢呋喃为溶剂时可以有 较好的收率. 在优化条件下, 我们对催化剂回收套用情 况进行实验, 具体实验结果如表 3 . 通过 5 次的回收套 用实验表明，腐殖酸催化剂活性并未有明显下降，说明

表 3 催化剂回收套用情况 ${ }^{a}$

Table 3 Results of catalyst recycle applied

\begin{tabular}{lllllll}
\hline 循环次数 & 0 & 1 & 2 & 3 & 4 & 5 \\
\hline 产率/\% & 95 & 95.5 & 95.5 & 94.5 & 95 & 94.5
\end{tabular}

${ }^{a} 1.0 \mathrm{mmol} \mathrm{4}$-甲氧基苯甲醛, $2.1 \mathrm{mmol}$ 乙酰乙酸乙酯, $0.1 \mathrm{~g}$ 腐殖酸, 2.0 $\mathrm{mmol}$ 醋酸铵, $10 \mathrm{~mL} 95 \%$ 乙醇, 回流 $4 \mathrm{~h}$.
腐殖酸催化剂回收使用效果较好.

我们进一步将腐殖酸原样和回收的腐殖酸进行 $\mathrm{X}$ 射线粉末衍射(PXRD)和透射电子显微镜(TEM)检测分 析, 如图 2 和图 3. 通过对比可知, 两者粉末衍射位置和 强度几乎是完全一致的，这说明催化剂在反应前后的结 构并没有变化; 透射电镜数据反映两者反应前后尺寸大 小基本一致，这说明在催化反应过程中，即使不断摚拌 催化剂的颗粒大小也没有发生变化. 更好的说明催化剂 可以重复利用.

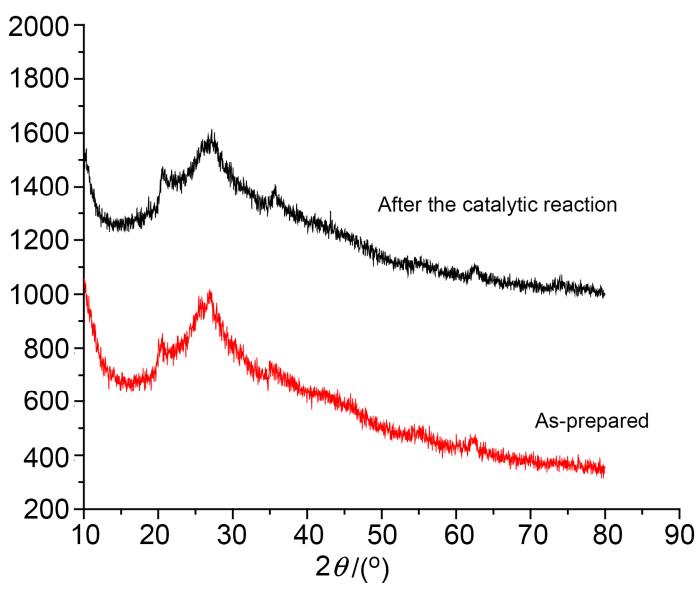

图 2 腐殖酸的粉末衍射图

Figure 2 PXRD patterns of humic caid

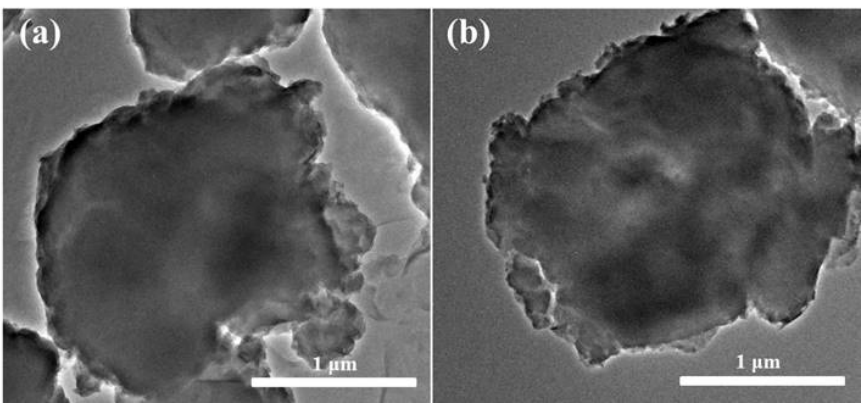

图 3 腐殖酸的投射电镜图

Figure 3 Tem patterns of humic caid (a) After the catalytic reaction; (b) As-prepared

此外我们对部分化合物进行放大 10 倍实验(表 4). 放大实验反应完全后, 我们将腐殖酸趁热过滤, 洗涤, 烘干回收, 滤液旋干回收乙醇, 用 $[V$ (乙醇 $): V($ 水 $)=$ $3: 1]$ 重结晶得到目标化合物.

\section{2 结论}

本文将腐殖酸用于催化有机合成反应. 报道了腐殖 酸催化醛、1,3-二羰基化合物和乙酸铵合成 1,4-二氢吡 啶类化合物. 方法操作方法简单、后处理方便、催化剂 廉价易得且能回收套用. 为 1,4-二氢吡啶类化合物的合 成寻找到了一种简捷绿色的方法. 
表 4 腐殖酸催化合成 1,4-二氢吡啶类化合物 ${ }^{a}$

Table 4 Humic acid catalyzed synthesis of 1,4-dihydropyridines

\begin{tabular}{|c|c|c|c|c|c|c|}
\hline \multirow{2}{*}{ Entry } & \multirow{2}{*}{$\mathrm{R}^{1}$} & \multirow{2}{*}{$\mathrm{R}^{2}$} & \multirow{2}{*}{ Time/h } & \multirow{2}{*}{ Yield/\% } & \multicolumn{2}{|c|}{ m.p. $/{ }^{\circ} \mathrm{C}$} \\
\hline & & & & & Actual & Reference \\
\hline $3 \mathbf{3 a}$ & $\mathrm{OC}_{2} \mathrm{H}_{5}$ & $4-\mathrm{CH}_{3} \mathrm{OC}_{6} \mathrm{H}_{4}$ & 4.0 & 95 & $158 \sim 159$ & $157 \sim 159^{[11]}$ \\
\hline $3 \mathbf{b}$ & $\mathrm{OC}_{2} \mathrm{H}_{5}$ & $4-\mathrm{O}_{2} \mathrm{NC}_{6} \mathrm{H}_{4}$ & 5.5 & 94 & $128 \sim 129$ & $129 \sim 130^{[10]}$ \\
\hline $3 c$ & $\mathrm{OC}_{2} \mathrm{H}_{5}$ & $4-\mathrm{CH}_{3} \mathrm{C}_{6} \mathrm{H}_{4}$ & 4.5 & 95 & $137 \sim 139$ & $138 \sim 141^{[10]}$ \\
\hline 3d & $\mathrm{OC}_{2} \mathrm{H}_{5}$ & $4-\mathrm{BrC}_{6} \mathrm{H}_{4}$ & 4.0 & 97 & $160 \sim 161$ & $158 \sim 160^{[10]}$ \\
\hline $3 e$ & $\mathrm{OC}_{2} \mathrm{H}_{5}$ & $2,4-(\mathrm{Cl})_{2} \mathrm{C}_{6} \mathrm{H}_{3}$ & 5.0 & 95 & $147 \sim 149$ & $148 \sim 150^{[10]}$ \\
\hline $3 f$ & $\mathrm{OC}_{2} \mathrm{H}_{5}$ & $\left(\mathrm{CH}_{3}\right)_{2} \mathrm{NC}_{6} \mathrm{H}_{4}$ & 4.5 & 94 & $195 \sim 196$ & $198 \sim 199^{[10]}$ \\
\hline $3 g$ & $\mathrm{OC}_{2} \mathrm{H}_{5}$ & 4-HO-3- $\mathrm{CH}_{3} \mathrm{C}_{6} \mathrm{H}_{3}$ & 4.5 & 93 & $144 \sim 146$ & $145 \sim 148^{[10]}$ \\
\hline $3 \mathbf{h}$ & $\mathrm{OCH}_{3}$ & $4-\mathrm{CH}_{3} \mathrm{OC}_{6} \mathrm{H}_{4}$ & 3.5 & 96 & $183 \sim 185$ & $185 \sim 188^{[10]}$ \\
\hline $3 \mathbf{i}$ & $\mathrm{OCH}_{3}$ & $4-\mathrm{O}_{2} \mathrm{NC}_{6} \mathrm{H}_{4}$ & 5.0 & 95 & $168 \sim 169$ & $174 \sim 177^{[10]}$ \\
\hline $3 \mathbf{j}$ & $\mathrm{OCH}_{3}$ & $4-\mathrm{CH}_{3} \mathrm{C}_{6} \mathrm{H}_{4}$ & 4.5 & 96 & $176 \sim 177$ & $177 \sim 180^{[10]}$ \\
\hline $3 \mathbf{k}$ & $\mathrm{OCH}_{3}$ & 4- $\mathrm{BrC}_{6} \mathrm{H}_{4}$ & 3.5 & 98 & $200 \sim 201$ & $201 \sim 202^{[9]}$ \\
\hline 31 & $\mathrm{OCH}_{3}$ & $2,4-(\mathrm{Cl})_{2} \mathrm{C}_{6} \mathrm{H}_{3}$ & 4.5 & 96 & $188 \sim 190$ & $189 \sim 192^{[11]}$ \\
\hline
\end{tabular}

${ }^{a}$ Amplification experiment: 3a, time 5.5 h, yield $96.5 \%$; 3d, time: 5.5 h, yield $98.5 \%$; 3k, time: 5.0 h, yield $98.5 \%$; 31, time: 5.5 h, yield $97 \%$.

\section{3 实验部分}

\section{1 仪器与试剂}

WRS-1 型数字熔点仪(温度计未校正); ZF-II 型四用 紫外线分析仪(TLC); BRUKER D8 ADVANCE 型 X 射线 衍射仪(扫描电压电流分别为 $40 \mathrm{kV} 、 40 \mathrm{~mA}, \lambda=0.15418$ $\mathrm{nm}$ ，扫描范围 $2 \theta=10^{\circ} \sim 80^{\circ}$ ); $300 \mathrm{kV}$ 高分辨透射电子 显微镜(Tecnai，荷兰 FEI 公司); Bruker 核磁共振仪(TMS 为内标, $400 \mathrm{MHz}$ ); 所用试剂均为分析纯级, 腐殖酸购 于阿拉丁, 其中黄腐酸 $\geqslant 90 \%$.

\section{2 实验方法}

\subsubsection{1,4-二氢吡啶(3a)合成步骤}

将 4-甲氧基苯甲酲 $(0.136 \mathrm{~g}, 1 \mathrm{mmol}) 、 乙$ 酰乙酸乙酯 $(0.275 \mathrm{~g}, 2.1 \mathrm{mmol})$ 、醋酸铵 $(0.154 \mathrm{~g}, 2 \mathrm{mmol})$ 、腐殖酸催 化剂 $(0.1 \mathrm{~g})$ 和 $10 \mathrm{~mL} 95 \%$ 的乙醇加入 $25 \mathrm{~mL}$ 圆底烧瓶中, 加热回流, 薄层色谱(TCL)跟踪反应进程, $4 \mathrm{~h}$ 后反应结 束, 将腐殖酸趁热过滤, 催化剂用乙醇洗涤, 烘干回收 利用. 滤液合并旋干后, 分批加入 $15 \mathrm{~mL}$ 乙酸乙酯溶解, 分出乙酸乙酯, 无水硫酸钠干燥、抽滤、脱溶, 残余物 经柱层析 $[V$ (石油醚) $: V$ (乙酸乙酯 $)=10 ： 1]$ 分离得到 目标化合物 3a.

2,6-二甲基-4-(4-甲氧基苯基)-3,5-二乙氧羰基-1,4二氢吡啶(3a): ${ }^{1} \mathrm{H}$ NMR $\left(400 \mathrm{MHz}, \mathrm{CDCl}_{3}\right) \delta: 7.20 \sim 6.75$ $\left(\mathrm{m}, 4 \mathrm{H}, \mathrm{C}_{6} \mathrm{H}_{4}\right), 5.79(\mathrm{~s}, 1 \mathrm{H}, \mathrm{NH}), 4.94(\mathrm{~s}, 1 \mathrm{H}, \mathrm{CH}), 4.10$ (q, $\left.J=7.1 \mathrm{~Hz}, 4 \mathrm{H}, 2 \times \mathrm{CH}_{2}\right), 3.76\left(\mathrm{~s}, 3 \mathrm{H}, \mathrm{CH}_{3}\right), 2.32(\mathrm{~s}, 6 \mathrm{H}$, $\left.2 \times \mathrm{CH}_{3}\right), 1.23\left(\mathrm{t}, J=7.1 \mathrm{~Hz}, 6 \mathrm{H}, 2 \times \mathrm{CH}_{3}\right) ;{ }^{13} \mathrm{C} \mathrm{NMR}(100$ $\left.\mathrm{MHz}, \mathrm{CDCl}_{3}\right) \delta: 167.60,143.50,140.23,128.82,113.07$, 104.22, 59.56, 55.01, 38.63, 19.39, 14.15 .

2,6-二甲基-4-(4-硝基苯基)-3,5-二乙氧羰基-1,4-二 氢吡啶(3b): ${ }^{1} \mathrm{H}$ NMR $\left(400 \mathrm{MHz}, \mathrm{CDCl}_{3}\right) \delta: 8.17 \sim 7.39$ $\left(\mathrm{m}, 4 \mathrm{H}, \mathrm{C}_{6} \mathrm{H}_{4}\right), 5.69(\mathrm{~s}, 1 \mathrm{H}, \mathrm{NH}), 5.10(\mathrm{~s}, 1 \mathrm{H}, \mathrm{CH}), 4.10(\mathrm{q}$,
$\left.J=7.1 \mathrm{~Hz}, 4 \mathrm{H}, 2 \times \mathrm{CH}_{2}\right), 2.37\left(\mathrm{~s}, 6 \mathrm{H}, 2 \times \mathrm{CH}_{3}\right), 1.23(\mathrm{t}$, $\left.J=7.1 \mathrm{~Hz}, 6 \mathrm{H}, 2 \times \mathrm{CH}_{3}\right) ;{ }^{13} \mathrm{C}$ NMR $\left(100 \mathrm{MHz}, \mathrm{CDCl}_{3}\right) \delta$ : $166.99,144.47,128.87,123.26,103.28,59.97,40.14$, $19.66,14.24$.

2,6-二甲基-4-(4-甲基苯基)-3,5-二乙氧羰基-1,4-二 氢吡啶(3c): ${ }^{1} \mathrm{H}$ NMR $\left(400 \mathrm{MHz}, \mathrm{CDCl}_{3}\right) \delta: 7.18 \sim 7.02$ $\left(\mathrm{m}, 4 \mathrm{H}, \mathrm{C}_{6} \mathrm{H}_{4}\right), 5.63(\mathrm{~s}, 1 \mathrm{H}, \mathrm{NH}), 4.97(\mathrm{~s}, 1 \mathrm{H}, \mathrm{CH}), 4.10(\mathrm{q}$, $\left.J=7.0,4 \mathrm{H}, 2 \times \mathrm{CH}_{2}\right), 2.33\left(\mathrm{~s}, 6 \mathrm{H}, 2 \times \mathrm{CH}_{3}\right), 2.28(\mathrm{~s}, 3 \mathrm{H}$, $\left.\mathrm{CH}_{3}\right), 1.24\left(\mathrm{t}, J=7.0 \mathrm{~Hz}, 6 \mathrm{H}, 2 \times \mathrm{CH}_{3}\right) ;{ }^{13} \mathrm{C} \mathrm{NMR}(100$ $\left.\mathrm{MHz}, \mathrm{CDCl}_{3}\right) \delta: 167.96,144.41,144.01,135.50,128.63$, $127.34,103.86,50.82,38.69,20.90,19.40$.

2,6-二甲基-4-(4-溴苯基)-3,5-二乙氧羰基-1,4-二氢 吡啶(3d): ${ }^{1} \mathrm{H}$ NMR (400 MHz, $\left.\mathrm{CDCl}_{3}\right) \delta: 7.42 \sim 7.07$ (m, $\left.4 \mathrm{H}, \mathrm{C}_{6} \mathrm{H}_{4}\right), 5.61(\mathrm{~s}, 1 \mathrm{H}, \mathrm{NH}), 4.96(\mathrm{~s}, 1 \mathrm{H}, \mathrm{CH}), 4.10$ (q, $J=$ 7.1, $\left.3.9 \mathrm{~Hz}, 4 \mathrm{H}, 2 \times \mathrm{CH}_{2}\right), 2.34\left(\mathrm{~s}, 6 \mathrm{H}, 2 \times \mathrm{CH}_{3}\right), 1.23(\mathrm{t}$, $\left.J=7.1 \mathrm{~Hz}, 6 \mathrm{H}, 2 \times \mathrm{CH}_{3}\right),{ }^{13} \mathrm{C}$ NMR $\left(100 \mathrm{MHz}, \mathrm{CDCl}_{3}\right) \delta$ : $167.33,146.78,143.86,130.87,129.81,103.88,59.80$, $39.34,19.60,14.24$.

2,6-二甲基-4-(2,4-二氯苯基)-3,5-二乙氧羰基-1,4二氢吡啶(3e): ${ }^{1} \mathrm{H}$ NMR $\left(400 \mathrm{MHz}, \mathrm{CDCl}_{3}\right) \delta: 7.37 \sim 7.10$ $\left(\mathrm{m}, 3 \mathrm{H}, \mathrm{C}_{6} \mathrm{H}_{3}\right), 5.85(\mathrm{~s}, 1 \mathrm{H}, \mathrm{NH}), 5.35(\mathrm{~s}, 1 \mathrm{H}, \mathrm{CH}), 4.08(\mathrm{q}$, $\left.J=6.4 \mathrm{~Hz}, 4 \mathrm{H}, 2 \times \mathrm{CH}_{2}\right), 2.29\left(\mathrm{~s}, 6 \mathrm{H}, 2 \times \mathrm{CH}_{3}\right), 1.21(\mathrm{t}$, $\left.J=6.4 \mathrm{~Hz}, 6 \mathrm{H}, 2 \times \mathrm{CH}_{3}\right) ;{ }^{13} \mathrm{C}$ NMR $\left(100 \mathrm{MHz}, \mathrm{CDCl}_{3}\right) \delta$ : $167.32,144.16,144.02,133.07,132.39,131.97,128.76$, $126.87,103.34,59.72,37.26,19.40,14.20$.

2,6-二甲基-4-(4- $N, N$-二甲基胺基苯基)-3,5-二乙氧 羰基-1,4-二氢吡啶(3f): ${ }^{1} \mathrm{H}$ NMR $\left(400 \mathrm{MHz}, \mathrm{CDCl}_{3}\right) \delta$ : $7.25 \sim 6.54\left(\mathrm{~m}, 4 \mathrm{H}, \mathrm{C}_{6} \mathrm{H}_{4}\right), 5.54(\mathrm{~s}, 1 \mathrm{H}, \mathrm{NH}), 4.90(\mathrm{~s}, 1 \mathrm{H}$, $\mathrm{CH}), 4.10\left(\mathrm{q}, J=7.2 \mathrm{~Hz}, 4 \mathrm{H}, 2 \times \mathrm{CH}_{2}\right), 2.90(\mathrm{~s}, 6 \mathrm{H}$, $\left.2 \times \mathrm{CH}_{3}\right), 2.33\left(\mathrm{~s}, 6 \mathrm{H}, 2 \times \mathrm{CH}_{3}\right), 1.25(\mathrm{t}, J=7.2 \mathrm{~Hz}, 6 \mathrm{H}$, $\left.2 \times \mathrm{CH}_{3}\right) ;{ }^{13} \mathrm{C}$ NMR $\left(100 \mathrm{MHz}, \mathrm{CDCl}_{3}\right) \delta: 167.61,146.58$, 
$143.19,131.12,128.55,124.33,100.06,59.49,38.16$, $18.87,14.15$.

2,6-二甲基-4-(3-甲基-4-羟基苯基)-3,5-二乙氧羰基1,4-二氢吡啶 $(\mathbf{3 g}):{ }^{1} \mathrm{H} \mathrm{NMR}\left(400 \mathrm{MHz}, \mathrm{CDCl}_{3}\right) \delta: 6.86 \sim$ $6.75\left(\mathrm{~m}, 3 \mathrm{H}, \mathrm{C}_{6} \mathrm{H}_{3}\right), 5.66(\mathrm{~s}, 1 \mathrm{H}, \mathrm{NH}), 5.48(\mathrm{~s}, 1 \mathrm{H}, \mathrm{CH})$, 4.94 (s, 1H), 4.12 (q, $\left.J=7.1 \mathrm{~Hz}, 4 \mathrm{H}, 2 \times \mathrm{CH}_{2}\right), 3.84$ (s, 3H, $\left.\mathrm{CH}_{3}\right), 2.33\left(\mathrm{~s}, 6 \mathrm{H}, 2 \times \mathrm{CH}_{3}\right), 1.25(\mathrm{t}, J=7.1 \mathrm{~Hz}, 6 \mathrm{H}$, $\left.2 \times \mathrm{CH}_{3}\right) ;{ }^{13} \mathrm{C} \mathrm{NMR}\left(100 \mathrm{MHz}, \mathrm{CDCl}_{3}\right) \delta: 167.56,145.71$, $143.81,143.33,120.38,113.74,110.84,104.26,59.55$, $55.66,39.05,19.45,14.20$.

2,6-二甲基-4-(4-甲氧基苯基)-3,5-二甲氧羰基-1,4二氢吡啶(3h): ${ }^{1} \mathrm{H} \mathrm{NMR}\left(400 \mathrm{MHz}, \mathrm{CDCl}_{3}\right) \delta: 7.24 \sim 6.70$ $\left(\mathrm{m}, 4 \mathrm{H}, \mathrm{C}_{6} \mathrm{H}_{4}\right), 5.84$ (s, 1H, NH), 4.95 (s, 1H, CH), 3.76 (s, $\left.3 \mathrm{H}, \mathrm{CH}_{3}\right), 3.65\left(\mathrm{~s}, 6 \mathrm{H}, 2 \times \mathrm{CH}_{3}\right), 2.32\left(\mathrm{~s}, 6 \mathrm{H}, 2 \times \mathrm{CH}_{3}\right) ;{ }^{13} \mathrm{C}$ NMR $\left(100 \mathrm{MHz}, \mathrm{CDCl}_{3}\right) \delta: 168.00,157.84,143.86$, 139.84, 128.46, 113.28, 103.96, 55.01, 50.83, 38.32, 19.38 .

2,6-二甲基-4-(4-硝基苯基)-3,5-二甲氧羰基-1,4-二 氢吡啶 $(3 i):{ }^{1} \mathrm{H}$ NMR $\left(400 \mathrm{MHz}, \mathrm{CDCl}_{3}\right) \delta: 8.17 \sim 7.37(\mathrm{~m}$, $\left.4 \mathrm{H}, \mathrm{C}_{6} \mathrm{H}_{4}\right), 5.86(\mathrm{~s}, 1 \mathrm{H}, \mathrm{NH}), 5.11(\mathrm{~s}, 1 \mathrm{H}, \mathrm{CH}), 3.65(\mathrm{~s}, 6 \mathrm{H}$, $\left.2 \times \mathrm{CH}_{3}\right), 2.36\left(\mathrm{~s}, 6 \mathrm{H}, 2 \times \mathrm{CH}_{3}\right) ;{ }^{13} \mathrm{C} \mathrm{NMR}(100 \mathrm{MHz}$, $\left.\mathrm{CDCl}_{3}\right) \delta: 167.43,154.73,146.41,144.88,128.57,123.43$, 102.99, 51.12, 39.86, 19.60 .

2,6-二甲基-4-(4-甲基苯基)-3,5-二甲氧羰基-1,4-二 氢吡啶 $(\mathbf{3 j})$ : ${ }^{1} \mathrm{H}$ NMR (400 $\left.\mathrm{MHz}, \mathrm{CDCl}_{3}\right) \delta: 7.16 \sim 7.03$ $\left(\mathrm{m}, 4 \mathrm{H}, \mathrm{C}_{6} \mathrm{H}_{4}\right), 5.81(\mathrm{~s}, 1 \mathrm{H}, \mathrm{NH}), 4.98(\mathrm{~s}, 1 \mathrm{H}, \mathrm{CH}), 3.66(\mathrm{~s}$, $\left.6 \mathrm{H}, 2 \times \mathrm{CH}_{3}\right), 2.33\left(\mathrm{~s}, 6 \mathrm{H}, 2 \times \mathrm{CH}_{3}\right), 2.29\left(\mathrm{~s}, 3 \mathrm{H}, \mathrm{CH}_{3}\right) ;{ }^{13} \mathrm{C}$ NMR $\left(100 \mathrm{MHz}, \mathrm{CDCl}_{3}\right) \delta: 167.96,144.41,143.99$, 135.50, 128.64, 127.35, 103.88, 50.81, 38.70, 20.89, 19.40 .

2,6-二甲基-4-(4-溴苯基)-3,5-二甲氧羰基-1,4-二氢 吡啶(3k): ${ }^{1} \mathrm{H}$ NMR (400 MHz, $\left.\mathrm{CDCl}_{3}\right) \delta: 7.39 \sim 7.15(\mathrm{~m}$, $\left.4 \mathrm{H}, \mathrm{C}_{6} \mathrm{H}_{4}\right), 5.72(\mathrm{~s}, 1 \mathrm{H}, \mathrm{NH}), 4.97(\mathrm{~s}, 1 \mathrm{H}, \mathrm{CH}), 3.65(\mathrm{~s}, 6 \mathrm{H}$, $\left.2 \times \mathrm{CH}_{3}\right), 2.34\left(\mathrm{~s}, 6 \mathrm{H}, 2 \times \mathrm{CH}_{3}\right) ;{ }^{13} \mathrm{C} \mathrm{NMR}(100 \mathrm{MHz}$, $\left.\mathrm{CDCl}_{3}\right) \delta: 167.77,146.46,144.25,131.06,129.47,119.97$, 103.60, 51.01, 39.04, 19.57 .

\section{2,6-二甲基-4-(2,4-二氯苯基)-3,5-二甲氧羰基-1,4-} 二氢吡啶(3I): ${ }^{1} \mathrm{H}$ NMR (400 MHz, $\left.\mathrm{CDCl}_{3}\right) \delta: 7.36 \sim 7.11$ $\left(\mathrm{m}, 3 \mathrm{H}, \mathrm{C}_{6} \mathrm{H}_{4}\right), 5.79(\mathrm{~s}, 1 \mathrm{H}, \mathrm{NH}), 5.36(\mathrm{~s}, 1 \mathrm{H}, \mathrm{CH}), 3.61(\mathrm{~s}$, $\left.6 \mathrm{H}, 2 \times \mathrm{CH}_{3}\right), 2.31\left(\mathrm{~s}, 6 \mathrm{H}, 2 \times \mathrm{CH}_{3}\right) ;{ }^{13} \mathrm{C} \mathrm{NMR}(100 \mathrm{MHz}$, $\left.\mathrm{CDCl}_{3}\right) \delta: 167.63,144.45,144.14,133.03,131.99,131.97$, $128.78,127.09,103.44,50.72,36.96,19.27$.
辅助材料(Supporting Information) 化合物的 ${ }^{1} \mathrm{H}$ NMR 和 ${ }^{13} \mathrm{C}$ NMR 谱图. 这些材料可以免费从本刊网站 (http://sioc-journal.cn/)上下载.

\section{Referenes}

[1] Goldmann, S.; Stoltefuss, J. Angew. Chem., Int. Ed. 1991, 30, 1559.

[2] Lu, L.-L.; Xu, H.; Zhou, P.; Yu, F.-C. Chin. J. Org. Chem. 2016, 36, 2858 (in Chinese). (鲁玲玲, 许辉, 周攀, 余富朝, 有机化学, 2016, 36, 2858.)

[3] Soo-Jeong, C.; Joong-Heui, C.; Isak, I. Eur. J. Med. Chem. 2010, $45,2578$.

[4] Hilgroth, A.; Lilie, H. Eur. J. Med. Chem. 2003, 38, 495.

[5] Prashantha, K. B. R.; Pankaj, M.; Karthikeyan, E.; Ankur, B.; Suja, P. V. Med. Chem. Res. 2010, 19, 344.

[6] Samzadeh, K. A.; Shafaroodi, H.; Miri, R.; Mirkhani, H. Med. Chem. Res. 2009, 18, 112.

[7] Kharkar, P. S.; Desai, B.; Gaveria, H. J. Med. Chem. 2002, 45 , 4858.

[8] Cooper, K.; Fray, M. J.; Parry, M. J. J. Med. Chem. 1992, 35, 3115.

[9] Nasr, M.; Hoseini, S.; Montazerozohori, M.; Mehrabi, R.; Nasrabadi, H. J. Mol. Catal. A: Chem. 2014, 382, 99.

[10] Bitaraf, M.; Ali, A.; Otokesh, S. J. Chin. Chem. Soc. 2016, 63, 336.

[11] Tamaddon, F.; Ghazi, S. Catal. Commun. 2015, 72, 63.

[12] Guo, S.-R.; Yuan, Y.-Q.; Zhang, C.-N.; Wu, X.-M.; Sun, C. Chin. J. Org. Chem. 2010, 28, 811 (in Chinese).

(郭圣荣，袁艳琴，张春牛，吴香梅，孙晨，有机化学，2010，28， 811.)

[13] Li, J.-P.; Chou, J.-K.; Li, H.-J.; Zhang, G.-S. Chin. J. Org. Chem. 2011, 29, 511 (in Chinese). (李建平, 仇记宽, 李会娟, 张贵生, 有机化学, 2011, 29, 511.)

[14] Wang, D.-L.; Dong, Z.; Liu Z.; Zhao W.; Yang, F.-F. Chin. J. Org. Chem. 2014, 34, 783 (in Chinese). (王道林，董哲，刘忠，赵伟，杨菲菲，有机化学, 2014, 34, 783.)

[15] Pei, W.; Wang, Q.; Li, X.-N.; Sun, C. Chin. J. Chem. 2010, 28, 483.

[16] Nikoorazm, M.; Ghorbani, C. A.; Khanmoradi, M. RSC Adv. 2016, 6,56549 .

[17] Ananda, K. T.; Mohan, C. V. S.; Satyanarayana, K. Synth. Commun. 2014, 44, 574.

[18] Tabassum, S.; Govindaraju, S.; Khan, R.; Pasha, M. A. RSC Adv. 2016, 6, 29802.

[19] Khaskel, A.; Barman, P. Heteroat. Chem. 2016, 27, 114.

[20] Zhang, Z.; Zeng, X.; Xie, D.; Chen, D.; Ding, L,; Wang, A.; Yang, L.; Zhong, G. Org. Lett. 2015, 17, 5052.

[21] Jiang, Y.-H.; Yan, C.-G. Chin. J. Chem. 2016, 34, 1255.

[22] Lin, W.; Zheng, Y.-X.; Huang, Z.-B.; Shi, D.-Q. Chin. J. Org. Chem. 2017, 37, 508 (in Chinese). (林伟，郑永祥，黄志斌，史达清，有机化学, 2017, 37, 508.)

[23] Shabalala, N.; Maddila, S.; Jonnalagadda, S. B. New J. Chem. 2016, 40, 5107.

[24] Kumar, A.; Sharma, S. Green Chem. 2011, 13, 2017.

[25] Wang, R.; Liu, X.; Wu, R.; Yu, B.; Li, H. RSC Adv. 2016, 6, 11.

[26] Xu, Q.-J.; Zhou, D.-P.; Cui, Y.-C. Chin. J. Org. Chem. 2007, 27, 1520 (in Chinese). (徐启杰, 周大鹏, 崔元臣, 有机化学, 2007, 27, 1520.) 